Pacific Journal of Mathematics

NOTE ON STOCHASTIC METHODS IN CONNECTION WITH
APPROXIMATION THEOREMS FOR POSITIVE LINEAR
OPERATORS 


\title{
A NOTE ON STOCHASTIC METHODS IN CONNECTION WITH APPROXIMATION THEOREMS FOR POSITIVE LINEAR OPERATORS
}

\author{
L. HAHN
}

In this paper some general approximation theorems in probability theory are used in order to deduce assertions on rates of convergence for several concrete positive linear operators, defined on the space of real continuous functions, towards the identity. Similar results are also established on the approximation of such operators towards an operator connected with the Gaussian distribution.

1. Intoduction. Any problem concerning positive linear operators on $C(J)$, the space of continuous real-valued functions defined on an interval $J \subset \boldsymbol{R}$, can naturally be interpreted as a problem in probability. A real random variable (r.v.) $Y$ or r.v. $Y_{n}$ are associated to the positive, linear operators $L, L_{n}: C(J) \rightarrow C(J), n \in N$, by setting

$$
L f(t):=E(f \circ Y) ; \quad L_{n} f(t):=E\left(f \circ Y_{n}\right)
$$

for fixed $t \in J, E(Y)$ denoting the expectation of $Y$ (see e.g., [9], [10], [11]). Apart from [8] which is restricted to pure convergence assertions, apparently all papers on this subject have in common the fact that the structure of the r.v. $Y, Y_{n}$ is not described any closer, although in the applications one uses that the r.v. are normalized sums.

The starting point of this paper are sequences of independent, not necessarily identically destributed r.v. $\left(X_{i}\right)_{i \in N}$ (defined on a common probability space $(\Omega, \mathscr{A}, P)$ ) with distribution $P_{X_{i}}(B)$ : = $P\left(\left\{\omega \in \Omega ; X_{i}(\omega) \in B\right\}\right)$, where $B$ is a Borel set of $\boldsymbol{R}$, variance $\operatorname{Var}\left(X_{i}\right)$ and distribution function (d.f.) $F_{X_{i}}(x):=P_{X_{i}}((-\infty, x]), x \in \boldsymbol{R}$. The aim is to study the convergence behavior of the normalized sums $\varphi(n) S_{n}$, where $S_{n}:=\sum_{i=1}^{n} X_{i}$, and $\varphi$ is an arbitrary normalizing function $\varphi: N \rightarrow\{x \in R, x>0\}=R^{+} \backslash\{0\} \quad$ with $\varphi(n)=o(1), \quad n \rightarrow \infty$, towards different limiting r.v., namely $X_{0}$ : $=0$ a.s. and $X^{*}$ (see page 9 for the definition). Examining the convergence towards the first r.v. $X_{0}$ corresponds, from the point of view of approximation theory, to the more important case of convergence of the operators $L_{n}$ towards $I$. It will turn out to be rather advantageous, to consider normalized sums $\varphi(n) S_{n}$ instead of arbitrary r.v. $Y_{n}$ since, on the one hand, this will not lead to any restrictions in the applications. On the other hand, it is now possible to describe the 
rate of convergence of the normalizing function $\varphi$, i.e., to give estimates in the form $\mathscr{O}\left(\varphi(n)^{\alpha}\right)$ for some $\alpha \in \boldsymbol{R}^{+}$.

Although the probability theorems to be presented are very general in the sense that each specialization of the r.v. $X_{i}$ results in a positive linear operator $L_{n}$, they actually arise from more general theorems of probability established in P. L. Butzer-L. Hahn $[2,3]$. If one looks at these papers, it is easy to see that convergence towards the limiting r.v. $X_{0}$ corresponds to the weak law of large numbers, and towards the r.v. $X^{*}$ to the central limit theorem. Since arbitrarily many operators may be obtained by specializing the r.v. $X_{i}$, one is forced to make a selection. The examples can be classified according to whether discrete or absolutely continuous r.v. are considered. In the former case one obtains, for example, the Bernstein, Szasz-Mirakjan and Baskakov operators; in the latter case, for example, the Weierstrass operator and two operators which arise from the gamma distribution. Several of these applications do not seem to have been considered so far.

Specifically, one obtains for the Bernstein operators

$$
B_{n} f(t):=\sum_{k=0}^{n} f\left(\frac{k}{n}\right)\left(\frac{n}{k}\right) t^{k}(1-t)^{n-k} \quad(f \in C[0,1], t \in[0,1])
$$

by taking the r.v. $X_{i}$ to be Bernoulli distributed, the estimate (cf. Corollary 2)

$$
\left|B_{n} f(t)-f(t)\right| \leqq M \omega_{2}\left(\left[\frac{t(1-t)}{n}\right]^{1 / 2}, f ; C[0,1]\right),
$$

$\omega_{2}(f, t ; C[0,1])$ being the second order of continuity of $f \in C[0,1]$. Apart from the constant $M$ this estimate is best possible (compare [4, p. 267]). If, on the other hand, one assumes the r.v. $X_{i}$ to be absolutely continuously distributed, e.g., normally distributed with mean 0 and variance $1 / 2$, then one has for the Weierstrass operator

$$
W_{n} f(t):=\sqrt{n / \pi} \int_{-\infty}^{\infty} f(x) \exp \left[-n(t-x)^{2}\right] d x,
$$

where $f=f_{1}+f_{2}$ with $f_{1} \in C_{b}(\boldsymbol{R}), f_{2} \in C_{b}^{2}(\boldsymbol{R})$ (for the definition see $\S 2$ ) the estimate (cf. Cor. 4)

$$
\left|W_{n} f(t)-f(t)\right| \leqq M \omega_{2}\left(1 / \sqrt{2 n}, f ; C_{b}(\boldsymbol{R})+C_{b}^{2}(\boldsymbol{R})\right) .
$$

Also the estimates to be established below for the Szász-Mirakjan and Baskakov operators are, apart from the constants occuring, best possible as M. Becker [1] has recently shown. This is indeed 
surprising since the results for these operators are deduced from very general encompassing theorems.

Section 2 is concerned with questions of notations, the general moduli of continuity and Lipschitz classes. In $\S 3$ general convergence theorems with rates are established, their applications being given in $\S 4$. In the following section, convergence assertions of these $L_{n}$ towards the associated operator of the r.v. $X^{*}$ are presented. An example of an operator constructed from a sequence of not identically distributed r.v. is given in the last $\S 6$.

The author would like to thank Professor P. L. Butzer for his critical reading of the manuscript and for valuable comments.

This work was supported by research grant No. II B2-FA 7233 of "Der Minister für Wisserschaft und Forschung des Landes Nordrhein Westfalen".

2. Notations and preliminaries. Let $C_{b}(J)$ the subset of $C(J)$ consisting of all bounded and uniformly continuous functions on $J$, endowed with norm $\|f\|:=\sup _{x \in J}|f(x)|$. For $r \in \boldsymbol{P}:=\{0,1,2, \cdots\}$ set

$$
C_{b}^{r}(J)=\left\{f \in C(J) ; f^{\prime}, f^{\prime \prime}, \cdots, f^{(r-1)} \in C(J), f^{(r)} \in C_{b}(J)\right\} .
$$

For any $f$ belonging to the algebraic sum

$$
C_{b}(J)+C_{b}^{r}(J):=\left\{f \in C(J) ; f=f_{1}+f_{2}, f_{1} \in C_{b}(J), f_{2} \in C_{b}^{r}(J)\right\}
$$

and any $t \geqq 0$, the $r$ th modulus of continuity is defined by

$$
\omega_{r}\left(t ; f ; C_{b}(J)+C_{b}^{r}(J)\right):=\sup _{|h| \leqq t}\left\|\sum_{k=0}^{r}(-1)^{r-k}\left(\begin{array}{l}
r \\
k
\end{array}\right) f(u+k h)\right\| .
$$

Lipschitz classes of index $r$ and of order $0<\alpha \leqq r$ are defined as usual by

$$
\begin{gathered}
\operatorname{Lip}\left(\alpha, r ; C_{b}(J)+C_{b}^{r}(J)\right):=\left\{f \in C_{b}(J)+C_{b}^{r}(J) ;\right. \\
\left.\omega_{r}\left(t, f ; C_{b}(J)+C_{b}^{r}(J)\right) \leqq L_{f} t^{\alpha}\right\} .
\end{gathered}
$$

For the sake of brevity, we will write $\omega_{r}(t, f)$ and $\operatorname{Lip}(\alpha, r)$, for (2.1) and (2.2), respectively. Furthermore we set $\widetilde{C}_{b}(J)$ : = $C_{b}(J)+C_{b}^{2}(J)$.

3. General convergence theorems with rates. We begin with two general theorems for sequences of real, independent r.v.

THEOREM 1. If 


$$
E\left(X_{i}^{2}\right)<\infty \quad(i \in N),
$$

then one has for any $f \in \widetilde{C}_{b}(R)$

$$
\begin{aligned}
& \left|\int_{-\infty}^{\infty} f(x) d F_{\varphi(n) S_{n}}(x)-f\left(\varphi(n) \sum_{i=1}^{n} E\left(X_{i}\right)\right)\right| \\
& \leqq M \omega_{2}\left(\left[\varphi(n)^{2} \sum_{i=1}^{n} \operatorname{Var}\left(X_{i}\right)\right]^{1 / 2}, f\right),
\end{aligned}
$$

the constant $M$ being independent of $f, n$ and $X_{i}, i \in N$. In particular, if $f \in \operatorname{Lip}(\alpha, 2), 0<\alpha \leqq 2$, then

$$
\begin{aligned}
& \left|\int_{-\infty}^{\infty} f(x) d F_{\varphi(n) S_{n}}(x)-f\left(\varphi(n) \sum_{i=1}^{n} E\left(X_{i}\right)\right)\right| \\
& \leqq M L_{f} \varphi(n)^{\alpha}\left[\sum_{i=1}^{n} \operatorname{Var}\left(X_{i}\right)\right]^{\alpha / 2} .
\end{aligned}
$$

Proof. We consider the sequence $\left(X_{i}^{\prime}\right)_{i \in N}$ of r.v. $X_{i}^{\prime}:=X_{i}$ $E\left(X_{i}\right)$, leading to $E\left(X_{i}^{\prime}\right)=E\left(X_{0}\right)=0$, and deduce by Theorem 4 in [2] (case $r=2$ ) that for $f \in \widetilde{C}_{b}(\boldsymbol{R})$ there exists a constant $M>0$ such that

$$
\begin{gathered}
\sup _{y \in \boldsymbol{R}}\left|\int_{-\infty}^{\infty} f(x+y) d F_{\varphi(n)\left[S_{n}-E\left(S_{n}\right)\right]}(x)-f(y)\right| \\
\leqq M \omega_{2}\left(\left[\varphi(n)^{2} \sum_{i=0}^{n} E\left(\left|X_{i}^{\prime}\right|^{2}\right)\right]^{1 / 2}, f\right) .
\end{gathered}
$$

Setting $y=y_{n}:=\varphi(n) E\left(S_{n}\right)$, this reads to (3.2) since

$$
\int_{-\infty}^{\infty} f\left(x+y_{n}\right) d F_{\varphi(n) S_{n}-y_{n}}(x)=\int_{-\infty}^{\infty} f(x) d F_{\varphi(n) S_{n}}(x) .
$$

If one would try to apply Theorem 4 in [2] for $r>2$, say $r=3$, in order to try to deduce higher order results, this would lead to the assumption

$$
\operatorname{Var}\left(X_{i}\right)=\int_{-\infty}^{\infty}\left[x-E\left(X_{i}\right)\right]^{2} d F_{X_{i}}(x)=\int_{-\infty}^{\infty} x^{2} d F_{X_{0}}(x)=0 .
$$

This would mean that $X_{i}=E\left(X_{i}\right)$ a.s., moreover $\varphi(n) S_{n}=$ $\varphi(n) \sum_{i=1}^{n} E\left(X_{i}\right)$ a.s., and so

$$
\int_{-\infty}^{\infty} f(x) d F_{\varphi(n) S_{n}}(x) \equiv f\left(\varphi(n) \sum_{i=1}^{n} E\left(X_{i}\right)\right),
$$

implying that the assertions would become trivial. Nevertheless one can weaken assumption (3.1) to obtain the following

THEOREM 2. Let $\beta \in(1,2]$ be such that

$$
E\left(\left|X_{i}\right|^{\beta}\right)<\infty \quad(i \in N) .
$$


Then for any $f \in \operatorname{Lip}(\beta, 2) \cap C_{b}(\boldsymbol{R})$

$$
\begin{aligned}
& \left|\int_{-\infty}^{\infty} f(x) d F_{\varphi(n) S_{n}}(x)-f\left(\varphi(n) E\left(S_{n}\right)\right)\right| \\
& \quad \leqq \frac{L_{f}}{2} \varphi(n)^{\beta} \sum_{i=1}^{n} E\left(\left|X_{i}-E\left(X_{i}\right)\right|^{\beta}\right) .
\end{aligned}
$$

The proof follows from Theorem 3 in [2] similarly as did the previous proof from Theorem 4 in [2].

Taking into account that apparently all known operators which allow probabilistic interpretations arise from identically distributed r.v., let us formulate Theorems 1 and 2 under this restriction, choosing furthermore $\varphi(n)=n^{-1}$ :

CoRollary 1. Let $\left(X_{i}\right)_{i \in N}$ be distributed as $X$, and let $t \in \boldsymbol{R}$ be arbitrary.

(a) Assume that $E\left(X^{2}\right)<\infty$ as well as

$$
E(X)=t \text {. }
$$

Then one has for $f \in \widetilde{C}_{b}(\boldsymbol{R})$

$$
\left|\int_{-\infty}^{\infty} f(x) d F_{S_{n} / n}(x)-f(t)\right| \leqq M \omega_{2}\left(\left[\frac{\operatorname{Var}(X)}{n}\right]^{1 / 2}, f\right) .
$$

(b) If $E\left(|X|^{\beta}\right)<\infty$ and (3.6) holds, then for any $f \in \operatorname{Lip}(\beta, 2) \cap$ $C_{b}(\boldsymbol{R}) 1<\beta \leqq 2$,

$$
\left|\int_{-\infty}^{\infty} f(x) d F_{S_{n} / n}(x)-f(t)\right| \leqq \frac{L_{f}}{2} \frac{E\left(\left|X_{i}-t\right|^{\beta}\right)}{n^{\beta-1}} .
$$

Recall that $\operatorname{Var}(X)=E\left(X^{2}\right)-t^{2}$ in view of (3.6).

4. Limit theorems with rates for some discrete and absolute continuous distributions.

4.1. Discrete Distributions. In this section we wish to apply the general theorem to deduce convergence assertions for positive linear operators $L_{n}$ towards the identity operator $I$. Hereby we examine the pointwise convergence $L_{n} f(t)$ towards $f(t)$ for $t \in \boldsymbol{R}$, $f \in \widetilde{C}_{b}(\boldsymbol{R})$. More exactly, for each $t \in \boldsymbol{R}$ we construct sequences $\left(X_{i}\right)_{i \in N}$ of r.v. leading to sequence of associated operators $\left(L_{n}\right)_{n \in N}$ such that there holds convergence at this fixed point.

Let us start off with an arbitrary finite discrete distribution

$$
P_{X}=\sum_{j=1}^{m} a_{j} \varepsilon_{x_{j}}
$$


where $\varepsilon_{a}$ denotes the point measure for $a \in \boldsymbol{R}$.

THEOREM 3. Let $t \in \boldsymbol{R}, m \in N$ be arbitrary. Assume that the $2 m$ real numbers $a_{1}, \cdots, a_{m}, x_{1}, \cdots, x_{m}$ depending on $t$, fulfill the conditions

$$
\begin{aligned}
& \text { (i) } a_{j} \geqq 0, \quad 1 \leqq j \leqq m \\
& \text { (ii) } \sum_{j=1}^{m} a_{j}=1 \\
& \text { (iii) } \sum_{j=1}^{m} a_{j} x_{j}=t .
\end{aligned}
$$

Then $f \in \widetilde{C}_{b}(\boldsymbol{R})$ implies

$$
\begin{aligned}
& \left|\sum f\left(\frac{\sum_{j=1}^{m} \nu_{j} x_{j}}{n}\right) \frac{n !}{\nu_{1} ! \cdots \nu_{m} !} a_{1}^{\nu_{1}} \cdots a_{m}^{\nu_{m}}-f(t)\right| \\
& \leqq M \omega_{2}\left(\left[\frac{\sum_{j=1}^{m} a_{j} x_{j}^{2}-t^{2}}{n}\right]^{1 / 2}, f\right),
\end{aligned}
$$

where the sum is extended over all $\nu_{1}, \cdots, \nu_{m} \in \boldsymbol{P}$ with $\sum_{j=1}^{m} \nu_{j}=n$.

Proof. Setting $P_{X}=\sum_{j=1}^{m} a_{j} \varepsilon_{x_{j}}$, the assumption (4.1) guarantees that $P_{X}$ is a distribution of a r.v. $X$ with mean $E(X)=t$. Now let $\left(X_{i}\right)_{i \in N}$ be a sequence of independent identically distributed r.v., all distributed as $X$; this is always possible for a suitable probability space. Then $P_{S_{n}}=P_{X_{1}} * \cdots * P_{X_{n}}=\left(\sum_{j=1}^{m} a_{j} \varepsilon_{x_{j}}\right)^{n *}$, where " $n$ *" denotes the $n$th fold convolution. The multinomial theorem now yields

$$
P_{S_{n} / n}=\sum \frac{n !}{\nu_{1} ! \cdots \nu_{m} !} a_{1}^{\nu_{1}} \cdots a_{m}^{\nu_{m}} \varepsilon_{\left(\nu_{1} x_{1}+\cdots+\nu_{m} x_{m}\right) / n},
$$

where the sum is extended over all $\nu_{1}, \cdots, \nu_{m} \in \boldsymbol{P}$ with $\sum_{j=1}^{m} \nu_{j}=n$, so that an application of Corollary 1 completes the proof.

REMARK. Since

$$
F_{n^{-1}} \sum_{j=1}^{m} \nu_{j} x_{j}(x)=\left\{\begin{array}{l}
0, x<\min _{1 \leqq j \leqq m}\left\{x_{j}\right\}:=t_{0} \\
1, x \geqq \max _{1 \leqq j \leqq m}\left\{x_{j}\right\}:=t_{1}
\end{array}\right.
$$

for all $\nu_{1}, \cdots, \nu_{m} \in P$ with $\sum_{j=1}^{m} \nu_{j}=n$, the integral with respect to $P_{S_{n^{\prime} n}}$ yields no contribution outside the interval $\left[t_{0}, t_{1}\right]$, and hence

$$
\int_{-\infty}^{\infty} f(x) P_{S_{n} / n}(d x)=\int_{t_{0}}^{t_{1}} f(x) P_{S_{n} / n}(d x) \quad\left(f \in \widetilde{C}_{b}(\boldsymbol{R})\right) .
$$


Therefore one can replace the function class $\widetilde{C}_{b}(\boldsymbol{R})$ in Theorem 3 by $\widetilde{C}_{b}\left(\left[t_{0}, t_{1}\right]\right)=C\left(\left[t_{0}, t_{1}\right]\right)$.

As a special case of theorem 3 let us consider the Bernstein operators for $f \in C([0,1]), t \in[0,1]$, defined by (1.1). Then Theorem 3 yields at once an estimate for this operator when setting $m=2$, $a_{1}=t, a_{2}=1-t, x_{1}=1, x_{2}=0$ for $t \in[0,1]$.

CoRollary 2. For $f \in C[0,1]$ one hast he pointwise estimate (1.2).

As further applications of Corollary 1 we wish to investigate the Szász-Mirakjan as well as particular Baskakov operators. They are defined by

$$
\begin{gathered}
M_{n} f(t)=e^{-n t} \sum_{k=0}^{\infty} f\left(\frac{k}{n}\right) \frac{(n t)^{k}}{k !} \quad(t \in[0, \infty)), \\
V_{n} f(t)=\sum_{k=0}^{\infty} f\left(\frac{k}{n}\right)\left(\begin{array}{c}
n+k-1 \\
k
\end{array}\right) \frac{t^{k}}{(1+t)^{n+k}} \quad(t \in[0, \infty)),
\end{gathered}
$$

respectively. As before, both operators can be constructed from a sequence $\left(X_{i}\right)_{i \in N}$ of real independent, identically distributed r.v., distributed as $X$, where now $P_{X}$ in an infinite discrete distribution. For the construction of the Szász-Mirakjan and Baskakov operators we consider for $t \in[0, \infty)$

$$
P_{X}=e^{-t} \sum_{k=0}^{\infty} \frac{t^{k}}{k !} \varepsilon_{k}, \quad P_{x}=\sum_{k=0}^{\infty}\left(\frac{1}{1+t}\right)\left(\frac{t}{1+t}\right)^{k} \varepsilon_{k} .
$$

Well-known computations lead to

$$
P_{s_{n}}=e^{-n t} \sum_{k=0}^{\infty} \frac{(n t)^{k}}{k !} \varepsilon_{k}, \quad P_{S_{n}}=\sum_{k=0}^{\infty}\left(\begin{array}{c}
n+k-1 \\
k
\end{array}\right) \frac{1}{(1+t)^{n}}\left(\frac{t}{1+t}\right)^{k} \varepsilon_{k} .
$$

Since $E(X)=t$, in both cases, Corollary 1 yields at once

Corollary 3. For the Szász-Mirakjan and Baskakov operators there holds

(a) For $f \in \widetilde{C}_{b}([0, \infty))$ one has

$$
\left|M_{n} f(t)-f(t)\right| \leqq M \omega_{2}\left(\left[\frac{t}{n}\right]^{1 / 2}, f\right)
$$

(b) For $f \in \widetilde{C}_{b}([0, \infty))$ one has

$$
\left|V_{n} f(t)-f(t)\right| \leqq M \omega_{2}\left(\left[\frac{t+t^{2}}{n}\right]^{1 / 2}, f\right) .
$$

Note that the estimate in Corollary 2 actually holds uniformly 
in the whole interval $[0,1]$ (since $t-t^{2} \leqq 1 / 4$ ), and in Corollary 3 only uniformly in any compact interval $[0, b], b>0$.

The results for the specific operators in Corollary 3 are not new, although they seem to be formulated explicitely only in M. Beckr [1] and W. Dickmeis-R. J. Nessel [5], at least for $M_{n}$ and $V_{n}$.

4.2. Absolutely continuous distributions. Now we consider absolutely continuous r.v., namely those which have a density (function) $g$. We start off with a normally distributed r.v. but now with arbitrary mean and variance, defined by its density

$$
g(x)=\frac{1}{\sqrt{2 \pi \sigma^{2}}} e^{-(x-\tau)^{2} / 2 \sigma^{2}}
$$

with mean $\tau \in \boldsymbol{R}$ and variance $\sigma^{2}>0$. The associated normally distributed r.v. with $\tau=0$ and $\sigma^{2}=1$ is denoted by $X^{*}$. Then we obtain for the Weierstrass operator (1.3).

CoROLlaRY 4. For $f \in \widetilde{C}_{b}(\boldsymbol{R})$ the estimate (1.4) holds uniformly in $t \in \boldsymbol{R}$.

Proof. Let the r.v. $X$ be normally distributed with mean $\tau=t$ and variance $\sigma^{2}=1 / 2$. Then $S_{n}$ is normally distributed with mean $n t$ and variance $n / 2$, hence

$$
\int_{-\infty}^{\infty} f(x) d F_{S_{n} / n}(x)=\frac{1}{\sqrt{\pi n}} \int_{-\infty}^{\infty} f\left(\frac{x}{n}\right) e^{-(n t-x)^{2} / n} d x=W_{n} f(t) .
$$

Using Corollary 1 (a) the assertion follows immediately. Notice that the modulus of continuity above is independent of $t \in \boldsymbol{R}$, so that the assertion of Corollary 4 holds uniformly in $t$, in contrast to all other examples.

For the next examples let the density function be the gamma density defined by

$$
g(x)=\left\{\begin{array}{cc}
\frac{1}{\Gamma(\nu)} \gamma^{\nu} x^{\nu-1} e^{-\gamma x}, & x \geqq 0 \\
0 & , \quad x<0
\end{array}\right.
$$

for $\gamma>0$ and $\nu>0$, where $\Gamma(\nu):=\int_{0}^{\infty} x^{\nu-1} e^{-x} d x$. Similarly to the normally distributed case one has two prameters, so that many operators could be constructed. Let us consider two such operators. First we may choose $\nu=1$, obtaining the exponential density; this leads to the operator

$$
H_{n} f(t):=\frac{n^{n}}{(n-1) ! t^{n}} \int_{0}^{\infty} f(x)^{n-1} e^{-n x / t} d x \quad(t>0)
$$


which can be found in $[7$, p. 219], where, using the weak law of large numbers, it is shown that $H_{n}$ defines an uniform approximation process on every compact subinterval of $\boldsymbol{R}^{+}$. Z. Ditzian-C. P. May [6] considered this operator in connection with semi-groups.

Secondly, we may choose $\gamma=1$ in (4.7), so that the corresponding operator becomes

$$
G_{n} f(t):=\frac{n^{n t}}{\Gamma(n t)} \int_{0}^{\infty} f(x) x^{n t-1} e^{-n x} d t \quad(t>0) .
$$

For these two operators, the second perhaps being new, there holds

Corollary 5. For $f \in \widetilde{C}_{b}([0, \infty))$ and fixed $t>0$, one has

$$
\begin{aligned}
& \left|H_{n} f(t)-f(t)\right| \leqq M \omega_{2}\left(\frac{t}{\sqrt{n}}, f\right), \\
& \left|G_{n} f(t)-f(t)\right| \leqq M \omega_{2}(\sqrt{t / n}, f) .
\end{aligned}
$$

Proof. (a) For the r.v. $X$ with density $g$ defined by (4.7) with $\nu=1$, one has $E(X)=1 / \gamma$ and $\operatorname{Var}(X)=1 / \gamma^{2}, \gamma>0$. Choosing $\gamma=1 / t$, this yields $E(X)=t$. Furthermore, the sum $S_{n}$ has density (cf. $[7$, p. 10])

$$
g_{S_{n}}(x)=\frac{1}{t}\left(\frac{x}{t}\right)^{n-1} \frac{1}{(n-1) !} e^{-x / t} \quad(x \geqq 0) .
$$

Now it is a well-known fact that

$$
g_{S_{n} / n}(x)=n g_{S_{n}}(n x),
$$

so that

$$
\int_{-\infty}^{\infty} f(x) d F_{S_{n} / n}(x)=H_{n} f(t)
$$

Since $\operatorname{Var}(X)=t^{2}$, the proof of (a) is completed using Corollary 1(a). (b) Here let $X$ be a r.v. with density $g$ defined by (4.7) with $\gamma=1$, and $\nu=t$. Then $E(X)=\operatorname{Var}(X)=t$, and $S_{n}$ has density

$$
g_{S_{n}}(x)=\frac{1}{\Gamma(n t)} x^{n t-1} e^{-x} \quad(x \geqq 0),
$$

so that Corollary 1(a) together with (4.10) leads to the desired assertion.

As a last application of Corollary 1(a) let us construct another operator from a r.v. that is uniformly distributed over an interval, say $[0, a]$ for some $a>0$. Then $X$ has the rectangular density 


$$
g(x)= \begin{cases}1 / a, & 0 \leqq x \leqq a \\ 0, & \text { elsewhere }\end{cases}
$$

For a real function $f$ write $f_{+}(x)=\max \{f(x), 0\}$ for the positive part of $f$. With this notation the d.f. of the r.v. $X$ in question becomes $F_{X}(x)=\left(x_{+}-(x-a)_{+}\right) / a$, and the related operator turns out to be

$$
U_{n} f(t):=\frac{1}{(n-1) !}\left(\frac{k}{t}\right)^{n} \sum_{k=0}^{n}(-1)^{k}\left(\begin{array}{l}
n \\
k
\end{array}\right) \int_{0}^{n t / k} f\left(\frac{2 k u}{n}\right)(u-t)_{+}^{n-1} d u,
$$

which can be considered to be a convolution of $f$ with a $n$-fold iterated integral of the constant function. For this operator, which may not have been considered so far, there holds

CoRollary 6. If $f \in \widetilde{C}_{b}([0,2 t]), t>0$, one has

$$
\left|U_{n} f(t)-f(t)\right| \leqq M \omega_{2}\left(\frac{t}{\sqrt{3 n}}, f\right) .
$$

Proof. Let $X$ be a r.v. with density defined by (4.11) and $a=$ $2 t>0$. Then $E(X)=t$ and $\operatorname{Var}(X)=(2 t)^{2} / 12$. Moreover, $S_{n}$ has density

$$
g_{S_{n}}(x)=\frac{1}{(2 t)^{n}(n-1) !} \sum_{k=0}^{n}(-1)^{k}\left(\begin{array}{l}
n \\
k
\end{array}\right)(x-2 k t)_{+}^{n-1} \quad(0 \leqq x \leqq 2 n t),
$$

and by (4.10)

$$
\int_{-\infty}^{\infty} f(x) d F_{S_{n} / n}(x)=\frac{n}{(2 t)^{n}(n-1) !} \sum_{k=0}^{n}(-1)^{k}\left(\begin{array}{l}
n \\
k
\end{array}\right) \int_{0}^{2 t} f(x)(n x-2 k t)_{+}^{n-1} d x,
$$

which can be written as (4.12) under the substitution $x=2 k u / n$.

For $t<0$ it is of course possible to consider a r.v. $X$ with density $g(x)=-1 / a$ for $a \leqq x<0,=0$ elsewhere, for negative $a \in \boldsymbol{R}$, and to construct an analogous operator such that the assertion of Corollary 6 also holds for negative $t$.

Many further examples of positive linear operators that arise from a r.v. $X$ could be constructed so that Corollary 1(a) is applicable. For all examples presented here, the second moment of the r.v. $X$ exists. But if one considers a stably distributed r.v. with essential parameter $\gamma, 1<\gamma<2$, then Corollary 1 (a) does not apply but $1(\mathrm{~b})$ does since $E\left(|X|^{\beta}\right)<\infty$ for $\beta<\gamma$, but $E\left(|X|^{\gamma}\right)=\infty$, yielding an operator for which (3.8) holds. Using the representation of stably distributed r.v. (compare e.g., [7, p. 549]), this leads after similar computations as in the proof of the previous corollaries to 
CoROLlaRy 7. For $1<\beta<\gamma<2$, one has for any $f \in \operatorname{Lip}(\beta, 2) \cap$ $C_{b}(\boldsymbol{R})$

$$
\left|\int_{-\infty}^{\infty} f(x) g_{n, r}(t-x) d x-f(t)\right|=\mathcal{O}\left(n^{1-\beta}\right)
$$

uniformly in $t \in \boldsymbol{R}$, where

$$
g_{n, \gamma}(x):=\frac{-1}{\pi|x|} \sum_{k=1}^{\infty}(-1)^{k} \frac{n^{(2 k-1)(\gamma-1) / \gamma}|x|^{2 k-1}}{(2 k-1) !} \Gamma\left(\frac{2 k-1}{\gamma}+1\right) .
$$

5. Limiting operator connected with Gaussian distribution. In this section let us examine convergence of operators not against the identity but towards an operator $T$ which is connected with the Gaussian distribution, namely

$$
T f=\frac{1}{\sqrt{2 \pi}} \int_{-\infty}^{\infty} e^{-x^{2} / 2} f(x) d x
$$

Note that $T f$ is a constant, i.e., $T$ is a linear functional. While Theorem 1 was essentially a corollary of our version of the weak law of large numbers, the next assertions follow from the general Theorem 11 in [3] concerning the central limit theorem. For identically distributed r.v. it reads

THEOREM 4. Let $\left(X_{i}\right)_{i \in N}$ be distributed as a r.v. $X$ with $0<$ $\operatorname{Var}(X)<\infty$ and $E\left(|X|^{3}\right)<\infty$. Then for any $f \in C_{b}(\boldsymbol{R})+C_{b}^{3}(\boldsymbol{R})$

$$
\begin{aligned}
& \left|\int_{-\infty}^{\infty} f\left(\frac{x-n E(X)}{\sqrt{n \operatorname{Var}(X)}}\right) d F_{S_{n}}(x)-T f\right| \\
& \quad \leqq M \omega_{3}\left(n^{-1 / 6}\left\{E\left(\left|\frac{X-E(X)}{\sqrt{\operatorname{Var}(X)}}\right|^{3}\right)+E\left(\left|X^{*}\right|^{3}\right)\right\}^{1 / 3}, f\right) .
\end{aligned}
$$

To apply this apparently new result, one only has to compute the third absolute central moment of the r.v. in question. For the example of the Bernstein operators there holds:

Corollary 7. For $f \in C_{b}(\boldsymbol{R})+C_{b}^{3}(\boldsymbol{R})$ and $t \in(0,1)$, one has

$$
\begin{aligned}
\mid \sum_{k=0}^{n} f & \left(\frac{k-n t}{\sqrt{n t(1-t)}}\right)\left(\begin{array}{l}
n \\
k
\end{array}\right) t^{k}(1-t)^{n-k}-\frac{1}{\sqrt{2 \pi}} \int_{-\infty}^{\infty} f(x) e^{-x^{2 / 2}} d x \mid \\
& \leqq M \omega_{3}\left(n^{-1 / 6}\left\{\frac{(1-t)^{2}+t^{2}}{\sqrt{t(1-t)}}+\frac{4}{\sqrt{2 \pi}}\right\}^{1 / 3}, f\right) .
\end{aligned}
$$

Note that this corollary is actually the classical De MoivreLaplace theorem (for the binomial distribution) supplied with rates. 
6. Example of not identically distributed R. V. In any case, Corollary 1 and all concrete operators presented so far deal with sequences of identically distributed r.v. We want to close our paper with an operator which is constructed from not identically distributed r.v. $X_{i}$; this example shows why such operators have hardly been considered as yet. Although the r.v. are very simply distributed, it is not possible to give an explicit representation of the associated operator.

For $i \in N$ consider the independent r.v. $X_{i}$ with distribution

$$
P_{X_{i}}=t \varepsilon_{i}+(1-t) \varepsilon_{0} \quad(0 \leqq t \leqq 1) .
$$

Then

$$
\begin{aligned}
P_{S_{n}} & =\left[t \varepsilon_{1}+(1-t) \varepsilon_{0}\right] * \cdots *\left[t \varepsilon_{n}+(1-t) \varepsilon_{0}\right] \\
& =\sum_{k=1}^{n(n+1) / 2} \sum_{m=0}^{n} Q(k, m, n) t^{m}(1-t)^{n-m} \varepsilon_{k},
\end{aligned}
$$

where $Q(k, m, n), k, m, n \in N$, is the number of partitions of $k$ into exactly $m$ unequal parts which do not exceed $n$, and for $k=0$, $Q(0, m, n)=0$ for $m \neq 0,=1$ for $m=0, n \in N$. This follows by the formula

$$
\prod_{i=0}^{n}\left(1+a x^{i}\right)=1+\sum_{k=1}^{n(n+1) / 2} \sum_{m=1}^{n} Q(k, m, n) a^{m} x^{k} .
$$

Furthermore, one has $\sum_{i=1}^{n} E\left(X_{i}\right)=\sum_{i=1}^{n} i t=t n(n+1) / 2$, and choosing $\varphi(n)=2 /[n(n+1)]$, yields $\varphi(n) \sum_{i=1}^{n} E\left(X_{i}\right)=t$. Finally, $\operatorname{Var}\left(X_{i}\right)=$ $i^{2} t(1-t)$, so that $\sum_{i=1}^{n} \operatorname{Var}\left(X_{i}\right)=t(1-t) n(n+1)(2 n+1) / 6$. An application of Theorem 4 now yields

CoRollary 7. For $f \in C([0,1])$ one has

$$
\begin{aligned}
& \left|\sum_{k=0}^{n(n+1) \cdot 2} \sum_{m=0}^{n} f\left(\frac{2 k}{n(n+1)}\right) Q(k, m, n) t^{m}(1-t)^{n-m}-f(t)\right| \\
& \quad \leqq M \omega_{2}\left(\sqrt{\frac{2 t(1-t)(2 n+1)}{3 n(n+1)}}, f\right) .
\end{aligned}
$$

Notice that just as the Bernstein operator the new operator is an algebraic polynomial of degree $n$, and also the rate of convergence is the same.

Let us close this paper with the remark that it would also be possible to present all of the results given just in the form of pure convergence assertions (i.e., without rates). For this purpose one would apply the general Theorems 5, 13 and 14 of [2,3] for r.v. with " $o(1)$-rates" of convergence to various operators given here. 
ACKNOWLEDGMENT. The author is indebted to a referee who gave valuable comments especially in connection with Corollary 7 and made the paper more readable.

\section{REFERENCES}

1. M. Becker, Global approximatıon theorems for Szàsz-Mirakjan and Baskakov operators in polynomial weight spaces, Indiana Univ. Math. J., 27 (1978), 127-142.

2. P. L. Butzer and L. Hahn, General theorems on rates of convergence in distribution of random variables. I. General limit theorems, J. Multivariate Analysis, 8 (1978), 181-201.

3 . - General theorems on rates of convergence in distribution of random variables. II. Applications to the stable limit laws and weak law of large numbers, J. Multivariate Analysis, 8 (1978), 202-221.

4. R. A. DeVore, The Approximation of Continuous Functions by Positive Linear Operators, Lecture Notes in Math. No. 293, Springer, Berlin, 1972.

5. W. Dickmeis and R. J. Nessel, Classical approximation processes in connection with Lax equivalence theorems with orders, Acta Sci. Math. (Szeged), 40 (1978), 33-48.

6. Z. Ditzian and C. P. May, Saturation classes for exponential formulae of semi-groups of operators, Proceedings of the Symposium "Spline Functions and Approximation Theory" at the University of Alberta, Edmonton, May 29-June 1, 1972 (edited by A. Meir and A. Sharma), ISNM Vol. 21, Birkhäuser, Basel (1973), 83-99.

7. W. Feller, An Introduction to Probability Theory and its Applications, Vol. II, Wiley, New York-London-Sydney, 1966.

8. S. Karlin and Z. Ziegler, Iteration of positive approximation operators, J. Approximation Theory, 3 (1970), 310-339.

9. J. P. King, Probability and positive linear operators, Rev. Roumaine Math. Pures Appl., 20 (1975), 325-327.

10. D. D. Stancu, Use of probabilistic methods in the theory of uniform approximation of continous functions, Rev. Roumaine Math. Pures Appl., 14 (1969), 673-691.

11. L. I. Strukov and A. F. Timan, Mathematical expectation of continuous functions of random variables. Smoothness and variance, Sibirsk. Mat. Z., 18 (1977), 658-664 (Russian). Sibirian Math. J., 18 (1977), 469-474. (English translation).

Received April 18, 1978 and in revised form July 1, 1981.

Technological University of Aachen

Aachen, W. Germany 



\section{PACIFIC JOURNAL OF MATHEMATICS}

\section{EDITORS}

DONALD BABBITT (Managing Editor)

University of California

Los Angeles, CA 90024

Hugo Rossi

University of Utah

Salt Lake City, UT 84112

C. C. MOore and Arthur Agus

University of California

Berkeley, CA 94720
J. DugundJI

Department of Mathematics

University of Southern California

Los Angeles, CA 90007

R. FinN and J. MILGRAM

Stanford University

Stanford, CA 94305

\section{ASSOCIATE EDITORS}
R. ARENS
E. F, BECKENBACH
B. H. NeumanN
F. WOLF
K. YoSHIDA

\section{SUPPORTING INSTITUTIONS}

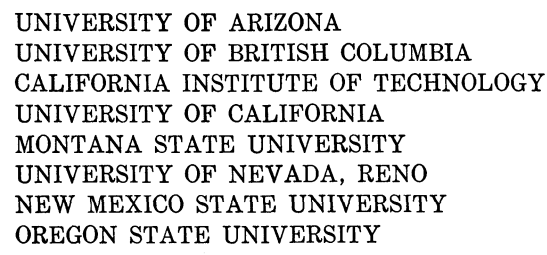

UNIVERSITY OF ARIZONA

UNIVERSITY OF BRITISH COLUMBIA

CALIFORNIA INSTITUTE OF TECHNOLOGY

UNIVERSITY OF CALIFORNIA

MONTANA STATE UNIVERSITY

UNIVERSITY OF NEVADA, RENO

NEW MEXICO STATE UNIVERSITY

OREGON STATE UNIVERSITY

\author{
UNIVERSITY OF OREGON \\ UNIVERSITY OF SOUTHERN CALIFORNIA \\ STANFORD UNIVERSITY \\ UNIVERSITY OF AAWAII \\ UNIVERSITY OF TOKYO \\ UNIVERSITY OF UTAH \\ WASHINGTON STATE UNIVERSITY \\ UNIVERSITY OF WASHINGTON
}

The Supporting Institutions listed above contribute to the cost of publication of this Journal, but they are not owners or publishers and have no responsibility for its content or policies,

Mathematical parers intended for publication in the Pacific Journal of Mathematics should be in typed form or offset-reproduced, (not dittoed), double spaced with large margins. Please do not use built up fractions in the text of the manuscript. However, you may use them in the displayed equations. Underline Greek letters in red, German in green, and script in blue. The first paragraph or two must be capable of being used separately as a synopsis of the entire paper. Please propose a heading for the odd unmbered pages of less than 35 characters. Manuscripts, in triplicate, may be sent to any one of the editors. Please classify according to the scheme of Math. Reviews, Index to Vol. 39. Supply name and address of author to whom proofs should be sent. All other communications should be addressed to the managing editor, or Elaine Barth, University of California, Los Angeles, California, 90024.

50 reprints to each author are provided free for each article, only if page charges have been substantially paid. Additional copies may be obtained at cost in multiples of 50 .

The Pacific Journal of Mathematics is issued monthly as of January 1966, Regular subscription rate: $\$ 114.00$ a year $(6$ Vol., 12 issues). Special rate: $\$ 57.00$ a year to individual members of supporting institution.

Subscriptions, orders for numbers issued in the last three calendar years, and changes of address shoud be sent to Pacific Journal of Mathematics, P.O. Box 969, Carmel Valley, CA 93924, U.S.A. Old back numbers obtainable from Kraus Periodicals Co., Route 100, Millwood, NY 10546.

PUBLISHED BY PACIFIC JOURNAL OF MATHEMATICS, A NON-PROFIT CORPORATION

Printed at Kokusai Bunken Insatsusha (International Academic Printing Co., Ltd.). 8-8, 3-chome, Takadanobaba, Shinjuku-ku, Tokyo 160, Japan.

Copyright (C) 1982 by Pacific Journal of Mathematics Manufactured and first issued in Japan 


\section{Pacific Journal of Mathematics}

\section{Vol. 101, No. 2 December, 1982}

Jean Bourgain, A Hausdorff-Young inequality for $B$-convex Banach

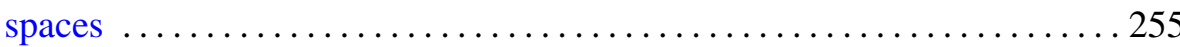

J. L. Brenner and Lorraine L. Foster, Exponential Diophantine

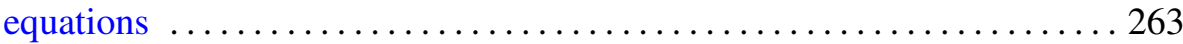

Henry H. Glover and William Duncan Homer, II, Fixed points on flag

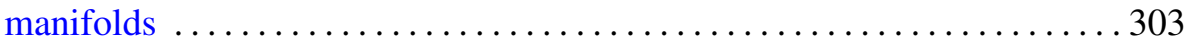

Lothar Hahn, A note on stochastic methods in connection with approximation theorems for positive linear operators $\ldots \ldots \ldots \ldots \ldots . \ldots 307$

James P. Henderson, Approximating cellular maps between low-dimensional polyhedra

V. K. Jain, Certain transformations of basic hypergeometric series and their

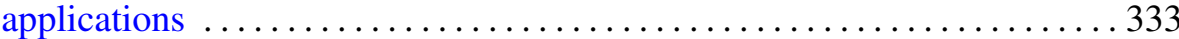

Charles David Keys, On the decomposition of reducible principal series

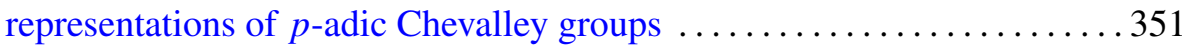

M. S. Klamkin and A. Meir, Ptolemy's inequality, chordal metric,

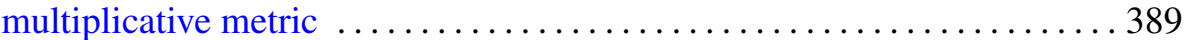

Robert F. Lax, Independence of normal Weierstrass points under

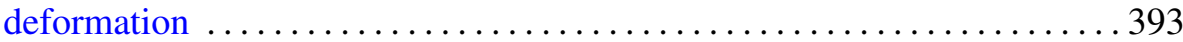

Leonid A. Luxemburg, On compactifications of metric spaces with

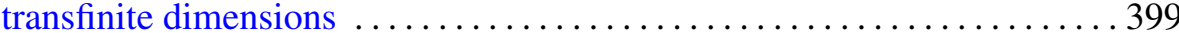

Carlton James Maxson, Martin Ross Pettet and Kirby C. Smith, On semisimple rings that are centralizer near-rings .....

Teodor C. Przymusiński, Extending functions from products with a metric factor and absolutes

Giorgio Talenti, A note on the Gauss curvature of harmonic and minimal surfaces

D. M. Terlinden, A spectral containment theorem analogous to the semigroup theory result $e^{t \sigma(A)} \subseteq \sigma\left(e^{t A}\right)$ 